\title{
Computed Tomography Attenuation Values of the High-Attenuating Crescent Sign Can Discriminate Between Rupture, Impending Rupture, and Non-Rupture of Aortic Aneurysms
}

\author{
Tatsuo Ueda, MD, PhD; Hiromitsu Hayashi, MD, PhD; Takahiro Ando, MD, PhD; \\ Kotomi Iwata, MD; Hidemasa Saito, MD, PhD; Shin-ichiro Kumita, MD, PhD
}

\begin{abstract}
Background: Although the high-attenuating crescent (HAC) sign can indicate aortic aneurysm (AA) impending rupture, the relation of its computed tomography (CT) value to the aneurysmal status remains unclear. This study compared the HAC sign CT-attenuation values among rupture, impending rupture, and non-rupture AA cases.

Methods and Results: This included 76 patients (mean age: 77.0 years) diagnosed with HAC sign-associated AA between January 2005 and July 2015. The CT-attenuation values of the HAC sign $(\mathrm{H})$ and aortic lumen $(\mathrm{A})$ using region-of-interest methodology were measured and the $H / A$ ratio was calculated. The study classified patients into the rupture group ( $R-G, n=36$ ), impending rupture group (IR-G, $n=16$ ), and non-rupture group (NR-G, $n=24$ ); the $H$ and the $H / A$ ratio were compared among them. Additionally, the $H$ and the $H / A$ ratio cut-offs between the IR-G and NR-G groups were evaluated. The $H$ and the $H / A$ ratio were significantly higher in the $R-G$ and IR-G than in the NR-G (both $P<0.001$ ); the $H / A$ ratio was significantly higher in the $R-G$ than in the IR-G $(P=0.038)$. The optimal cut-off for $\mathrm{H}$ between the IR-G and NR-G was 50.3 Hounsfield units (area under the curve $[A U C]=0.875$; sensitivity=87.5\%; specificity=87.5\%), and that for the $\mathrm{H} / \mathrm{A}$ ratio was 1.3 ( $\mathrm{AUC}=0.909$; sensitivity=91.7\%; specificity=87.5\%).
\end{abstract}

Conclusions: Among patients with $\mathrm{AA}$, the $\mathrm{H}$ and the $\mathrm{H} / \mathrm{A}$ ratio were significantly higher in cases of rupture and impending rupture than in those of non-rupture.

Key Words: Aortic aneurysm; Computed tomography (CT); CT-attenuation values; High-attenuating crescent sign; Impending rupture

$\mathbf{R}$ upture of an aortic aneurysm (AA) is associated with a high mortality rate. Although the diagnosis of a ruptured AA is usually straightforward, the detection of the signs of impending rupture can be challenging. The high-attenuating crescent (HAC) sign is regarded as an indicator of impending rupture or rupture of the abdominal AA. ${ }^{1-13}$ In addition, the HAC sign of a thoracic AA may represent an acute contained or impending rupture, analogous to that described in abdominal AA. ${ }^{14}$ This sign is defined as a crescent-shaped high-density area in the aortic wall or a mural thrombus on a computed tomography (CT) image. It appears as an intramural area with attenuation higher than that of the patent luminal region on unenhanced CT or higher than that of the psoas muscle on contrast-enhanced CT. ${ }^{15,16}$ The HAC sign in patients with a ruptured abdominal AA may be histopathologically attributed to acute hemorrhage in the mural thrombus or the aneurysmal wall. ${ }^{17}$ Roy et al reported that there was a tendency for AAs showing the crescent sign to be higher than those without the sign, although the difference was not found to be statistically significant. ${ }^{18}$ In a further study by Siegel et al, a crescent sign of high attenuation was present in $21 \%$ of cases of AA rupture, but not in cases of intact AAs. ${ }^{2}$ Thus, the HAC sign may be an indicator of impending rupture, particularly in patients with painful AAs. ${ }^{1}$ The overall sensitivity of the HAC sign, as an indicator of aneurysmal complication, was $77 \%$, with the specificity being $93 \%,{ }^{1}$ however, the positive predictive value of the sign was low $(53 \%){ }^{1}$ Therefore, it is occasionally difficult to distinguish impending rupture of an AA from nonrupture.1,19

Conversely, acute hemorrhage appears with a higher density than that observed for chronic hemorrhage on CT images. Therefore, we hypothesized that the HAC sign could indicate a higher density in cases of impending rupture or rupture compared to non-rupture cases. Indeed,

Received June 15, 2021; revised manuscript received September 11, 2021; accepted September 28, 2021; J-STAGE Advance Publication released online October 28, 2021 Time for primary review: 47 days

Department of Radiology, Nippon Medical School Hospital, Tokyo, Japan

Mailing address: Tatsuo Ueda, MD, PhD, Department of Radiology, Nippon Medical School Hospital, 1-1-5 Sendagi, Bunkyo-ku, Tokyo 113-8603, Japan. E-mail: s9015@nms.ac.jp

All rights are reserved to the Japanese Circulation Society. For permissions, please e-mail: cj@j-circ.or.jp

ISSN-1346-9843 


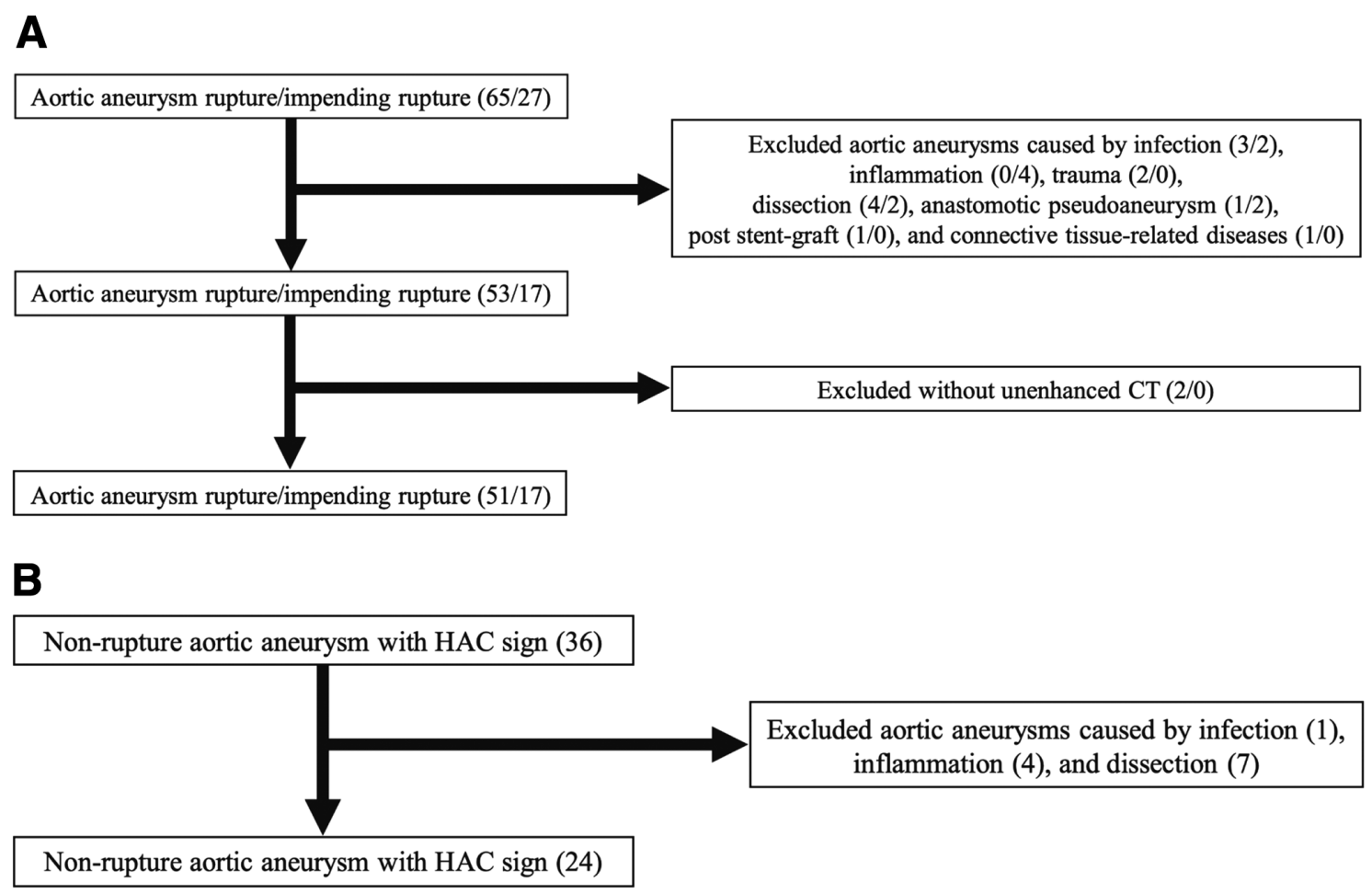

Figure 1. (A) The flowchart shows patient selection for AA rupture/impending rupture. (B) The flowchart shows patient selection for non-rupture with a HAC sign. AA, aortic aneurysm; CT, computed tomography; HAC, high-attenuating crescent.

Roy et al reported that the attenuation in the aortic aneurysmal thrombus was significantly higher in patients with rupture than in those with intact AAs; however, to the best of our knowledge, few reports to date have evaluated the CT attenuation value of the HAC sign, ${ }^{18}$ and ours is the first to compare the CT attenuation values of the HAC sign among cases of rupture, impending rupture, and nonrupture of AAs.

\section{Methods}

\section{Patients}

This study was approved by the institutional review board of the Nippon Medical School Hospital. The requirement for informed consent was waived because of the retrospective nature of the study. The authors had control of the data and information submitted for publication.

We reviewed the medical record system of our hospital from January 2005 to July 2015 searching for AA official CT reports conducted by certified diagnostic radiologists. Medical records were also reviewed for symptoms, comorbidities, causes of AAs, presence of the HAC sign, and subsequent AA rupture, surgical records, or both. We diagnosed aortic rupture/impending rupture according to the definitions described in a following section using these medical records. As a result, 92 consecutive patients were diagnosed with rupture/impending rupture (65/27) of AA. Among them, we excluded 22 patients with rupture/impending rupture $(12 / 10)$ caused by infection $(3 / 2)$, inflammation $(0 / 4)$, trauma (2/0), dissection (4/2), anastomotic pseudoaneurysm $(1 / 2)$, post stent-graft $(1 / 0)$, and connective tissue-related diseases (1/0), such as Marfan or Loeys-Dietz syndrome. Furthermore, we excluded 2 patients with rupture/impending rupture who did not undergo an unenhanced CT (2/0). Therefore, we included 68 patients with AA rupture/ impending rupture (51/17) (Figure 1A). Moreover, as a control group, we selected 36 consecutive patients from our hospital who were diagnosed with asymptomatic nonrupture AA with a HAC sign using CT reports conducted by certified diagnostic radiologists, between January 2005 and July 2015. By using medical records and CT imaging, we confirmed that no patient had pain related to the AA or experienced AA rupture for $>1$ month after the initial CT imaging. Among these participants, we excluded 12 non-rupture patients with a HAC sign caused by infection $(n=1)$, inflammation $(n=4)$, and dissection $(n=7)$. Therefore, we included 24 patients with non-rupture AA (Figure 1B).

\section{CT Imaging}

CT examinations were performed using a 320-row multidetector CT scanner (Aquilion ONETM; Canon Medical Systems, Tochigi, Japan), a 256-row multidetector CT scanner (Revolution CT; GE Healthcare, Chicago, IL, USA), 64-row multidetector CT scanners (GoldSeal Optima CT660 and LightSpeed VCT; GE Healthcare), a 32-row multidetector CT scanner (LightSpeed VCT; GE Healthcare), and a 16-row multidetector CT scanner (ECLOS, Hitachi, Tokyo, Japan). The detailed imaging parameters were as follows: tube voltage, $120 \mathrm{kV}$; tube current, $200-650 \mathrm{~mA}$ depending on the body mass index; gantry rotation speed, $0.35-0.4$ s/rotation depending on the CT scanner; collimation, $64 \times 0.5 \mathrm{~mm}$; and helical pitch, 0.984 . Helical data were 
Table 1. Clinical Characteristics of the Study Participants

\begin{tabular}{lc} 
& \multicolumn{1}{c}{ Characteristic } \\
Patients $(\mathrm{n})$ & 76 \\
Age (years, mean $\pm \mathrm{SD})$ & $77.0 \pm 9.9$ \\
Sex (male/female, $\mathrm{n} / \mathrm{n}$ ) & $53 / 23$ \\
Hypertension, $\mathrm{n}(\%)$ & $55(72.4)$ \\
Dyslipidemia, $\mathrm{n}(\%)$ & $26(34.2)$ \\
Diabetes mellitus, $\mathrm{n}(\%)$ & $9(11.8)$ \\
Location (thoracic/abdominal, $\mathrm{n} / \mathrm{n}$ ) & $28 / 48$ \\
Shape (fusiform/saccular, $\mathrm{n} / \mathrm{n}$ ) & $61 / 15$ \\
Diameter of the maximum minor axis & $62.0 \pm 14.8$ \\
(mm, mean $\pm \mathrm{SD}$ ) & \\
\hline
\end{tabular}

$\mathrm{SD}$, standard deviation.

reconstructed in the axial plane with $5-\mathrm{mm}$ section thickness and 5-mm intervals.

\section{Definitions of Ruptured, Impending Ruptured, and Non-Ruptured AAs}

A ruptured AA was defined as an AA with periaortic hemorrhage on a CT image. An AA with impending rupture was defined as an AA that ruptured within 1 week after the diagnosis using CT or if it satisfied the following criteria: (1) it was an AA with an HAC sign; (2) the patient experienced pain related to the AA; and (3) the AA was confirmed to be the sole cause of pain. An AA with non-rupture was defined as an AA that did not rupture within 1 month after the diagnosis using CT.

\section{Definition of the HAC Sign}

The HAC sign was defined as a crescent-shaped high-density or localized area with higher attenuation in the aortic wall or the mural thrombus than that in the aortic lumen on an unenhanced CT image.

\section{Measurements}

The HAC signs were all identified by certified diagnostic radiologists in their official $\mathrm{CT}$ reports. To quantify the level of attenuation in the HAC sign, we used CT software (We VIEW Z-edition; Hitachi) and measured the mean CT attenuation value, in Hounsfield unit (HU), of the HAC sign in an axial image. Two radiologists (T.U., 13 years of experience; T.A., 5 years of experience) manually measured the CT attenuation value of the aortic lumen and the HAC sign area slice on the same unenhanced CT slice using the region-of-interest methodology. The slice included the highest $\mathrm{CT}$ attenuation value of the HAC sign. The HAC sign area was measured only in the area where the CT value was relatively high. The mean $\mathrm{CT}$ attenuation values of the measurements were defined as the CT attenuation values of the aortic lumen (A) and the HAC sign (H). We calculated the $\mathrm{H} / \mathrm{A}$ ratio.

\section{Evaluations}

We evaluated the accuracy of the HAC sign in the rupture/ impending rupture cases. In addition, we classified AAs with a HAC sign into 3 groups as follows: rupture group (R-G), impending rupture group (IR-G), and non-rupture

\begin{tabular}{|c|c|c|c|c|c|c|}
\hline & \multirow{2}{*}{$\mathbf{R}-\mathbf{G}$} & \multirow{2}{*}{ IR-G } & \multirow{2}{*}{ NR-G } & \multicolumn{3}{|c|}{$P$ value } \\
\hline & & & & R-G vs. IR-G & IR-G vs. NR-G & R-G vs. NR-G \\
\hline Patients ( $n$ ) & 36 & 16 & 24 & & & \\
\hline Age (years, mean $\pm S D$ ) & $77.4 \pm 10.4$ & $77.8 \pm 11.3$ & $76.0 \pm 8.3$ & $0.992^{*}$ & $0.857^{*}$ & $0.866^{*}$ \\
\hline Sex (male/female, $n / n)$ & $25 / 11$ & $13 / 3$ & $15 / 9$ & $1^{\star \star}$ & $1^{\star *}$ & $1^{\star *}$ \\
\hline Hypertension, n (\%) & $28(87.5)$ & $11(91.7)$ & $16(84.2)$ & $1^{* *}$ & $1^{\star *}$ & $0.96^{\star *}$ \\
\hline Dyslipidemia, n (\%) & $11(35.5)$ & $6(60.0)$ & $9(47.4)$ & $1^{* *}$ & $1^{\star *}$ & $1^{\star *}$ \\
\hline Diabetes mellitus, n (\%) & $5(15.6)$ & $2(20.0)$ & $2(10.5)$ & $1^{* *}$ & $1^{* *}$ & $1^{* *}$ \\
\hline Hemoglobin $(\mathrm{g} / \mathrm{dL}$, mean $\pm \mathrm{SD})$ & $9.9 \pm 2.0$ & $12.1 \pm 2.0$ & $12.6 \pm 2.7$ & $0.005^{*}$ & $0.772^{*}$ & $<0.001^{*}$ \\
\hline Location (thoracic/abdominal, $\mathrm{n} / \mathrm{n}$ ) & $15 / 21$ & $3 / 13$ & $10 / 14$ & $0.39^{\star *}$ & $0.53^{\star \star}$ & $1^{* *}$ \\
\hline Shape (fusiform/saccular, n/n) & $26 / 10$ & $15 / 1$ & $20 / 4$ & $0.42^{\star \star}$ & $1^{\star *}$ & $1^{* *}$ \\
\hline $\begin{array}{l}\text { Diameter of the maximum minor axis } \\
(\mathrm{mm}, \mathrm{mean} \pm \mathrm{SD})\end{array}$ & $69.1 \pm 14.3$ & $62.8 \pm 12.6$ & $50.9 \pm 9.5$ & $0.232^{*}$ & $0.012^{*}$ & $<0.001^{*}$ \\
\hline
\end{tabular}

${ }^{*}$ One-way analysis of variance with Tukey's adjustment. ${ }^{* *}$ Chi-squared tests with Bonferroni adjustment. IR-G, impending rupture group; NR-G, non-rupture group; R-G, rupture group; SD, standard deviation.

\begin{tabular}{|c|c|c|c|c|c|c|}
\hline & \multirow{2}{*}{ R-G } & \multirow{2}{*}{ IR-G } & \multirow{2}{*}{ NR-G } & \multicolumn{3}{|c|}{$P$ value } \\
\hline & & & & R-G vs. IR-G & IR-G vs. NR-G & R-G vs. NR-G \\
\hline $\mathrm{H}(\mathrm{HU}$, mean $\pm \mathrm{SD})$ & $53.7 \pm 6.4$ & $55.5 \pm 5.7$ & $45.4 \pm 7.9$ & $0.636^{*}$ & $<0.001^{*}$ & $<0.001^{*}$ \\
\hline $\mathrm{A}(\mathrm{HU}$, mean $\pm \mathrm{SD})$ & $35.5 \pm 5.1$ & $40.5 \pm 4.4$ & $41.0 \pm 4.3$ & $0.002^{*}$ & $0.930^{*}$ & $<0.001^{*}$ \\
\hline $\mathrm{H} / \mathrm{A}$ ratio $($ mean $\pm \mathrm{SD})$ & $1.54 \pm 0.25$ & $1.38 \pm 0.16$ & $1.11 \pm 0.16$ & $0.038^{*}$ & $<0.001^{*}$ & $<0.001^{*}$ \\
\hline
\end{tabular}

${ }^{*}$ One-way analysis of variance with Tukey's adjustment. A, computed tomography (CT) attenuation value of the aortic lumen; $\mathrm{H}, \mathrm{CT}$ attenuation value of the high-attenuating crescent sign; HAC, high-attenuating crescent; IR-G, impending rupture group; NR-G, non-rupture group; HU, Hounsfield units; R-G, rupture group; SD, standard deviation. 


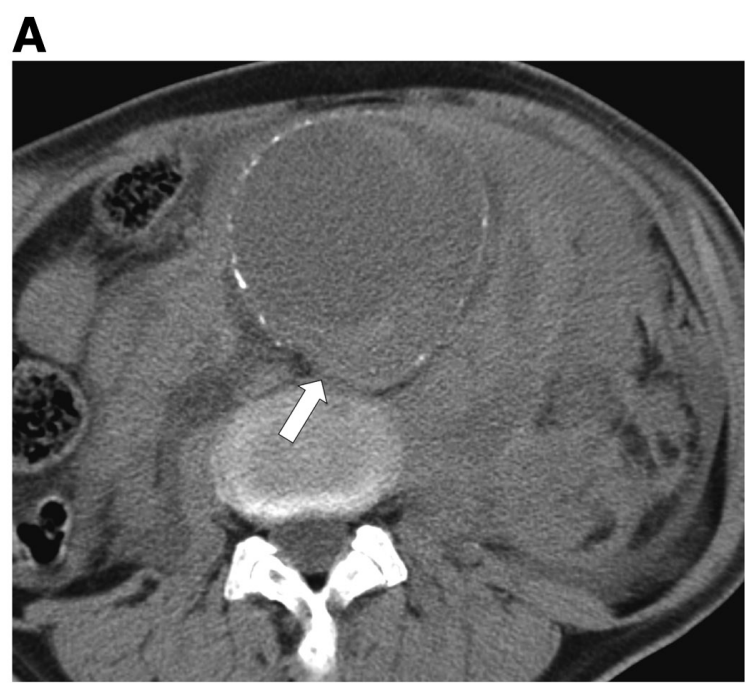

B

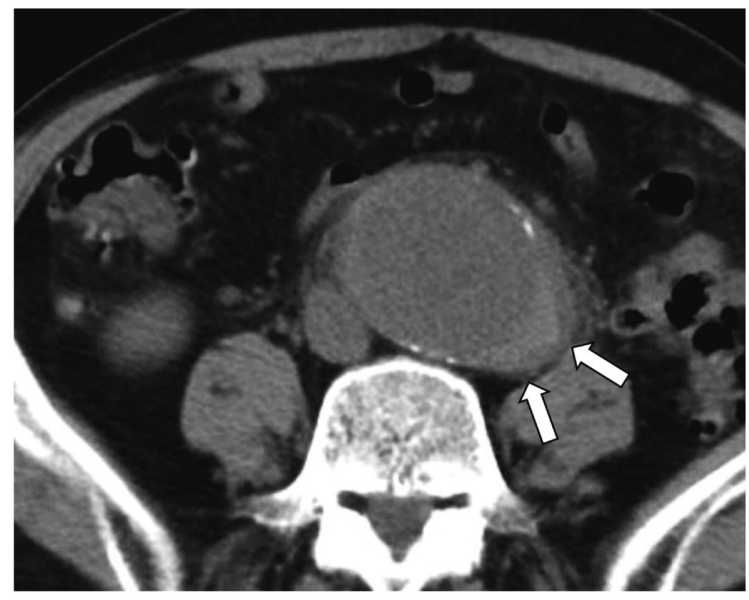

C

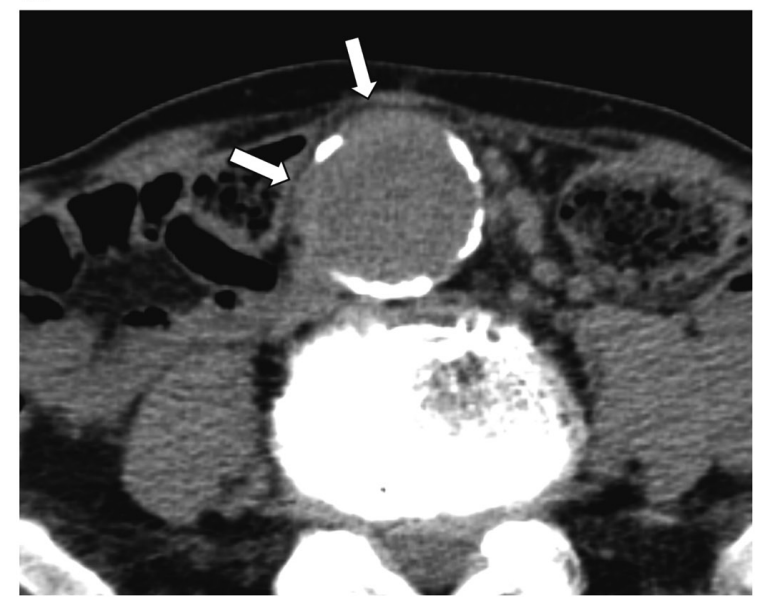

Figure 2. (A) Axial unenhanced CT image of an AA-rupture case. Unenhanced CT image of an AA with rupture in a 76-year-old man shows an HAC sign in the posterior abdominal aortic aneurysmal wall (arrow). The CT attenuation values of the $\mathrm{H}$ and the $\mathrm{A}$ were 53.8 and $34.7 \mathrm{HU}$, respectively, whereas the $\mathrm{H} / \mathrm{A}$ ratio was 1.56. (B) Axial unenhanced $\mathrm{CT}$ image of an $\mathrm{AA}$ with impending rupture. Unenhanced CT image of an AA with impending rupture in a 56-year-old man shows an HAC sign in the posterolateral abdominal aortic aneurysmal wall (arrow). The CT attenuation values of the $\mathrm{H}$ and the $\mathrm{A}$ were 60.7 and $38.7 \mathrm{HU}$, respectively, and the $\mathrm{H} / \mathrm{A}$ ratio was 1.57. (C) Axial unenhanced CT image of an AA with non-rupture. Unenhanced CT image of an unruptured AA in a 73-year-old man shows a HAC sign in the anterolateral abdominal aortic aneurysmal wall (arrow). The CT attenuation values of the $\mathrm{H}$ and the A were 52.3 and $47.2 \mathrm{HU}$, respectively, and the $\mathrm{H} / \mathrm{A}$ ratio was 1.11. A, computed tomography attenuation value of the aortic lumen; AA, aortic aneurysm; $\mathrm{CT}$, computed tomography; $\mathrm{H}$, computed tomography attenuation value of the highattenuating crescent sign; HAC, high-attenuating crescent; HU, Hounsfield unit.

group (NR-G). We compared the features of the AAs (location, shape, and diameter of the maximum minor axis), $\mathrm{H}$, and $\mathrm{H} / \mathrm{A}$ ratio among the 3 groups. Additionally, we evaluated the cut-off values of the $\mathrm{H}$ and $\mathrm{H} / \mathrm{A}$ ratio between the IR-G and NR-G.

\section{Statistical Analysis}

Continuous variables are presented as means and standard deviations. The R-G, IR-G, and NR-G were compared using chi-squared tests with Bonferroni and 1-way ANOVA with Tukey's honestly significant difference test for multiple comparisons. The receiver operator characteristic (ROC) curves of the $\mathrm{H}$ and $\mathrm{H} / \mathrm{A}$ ratio were constructed to identify the respective optimal cut-off values. SPSS Statistics version 19 for Windows (IBM Corp., Armonk, NY, USA) was used to perform all statistical analyses, and $\mathrm{P}$ values $<0.05$ indicated statistical significance.

\section{Results}

\section{Patient Characteristics}

The study included 68 patients having AA rupture/impending rupture (51/17) and 24 patients having non-rupture AA. There were 36 rupture and 16 impending rupture cases with a HAC sign, and 15 rupture and 1 impending rupture cases without a HAC sign. Therefore, the sensitiv- 

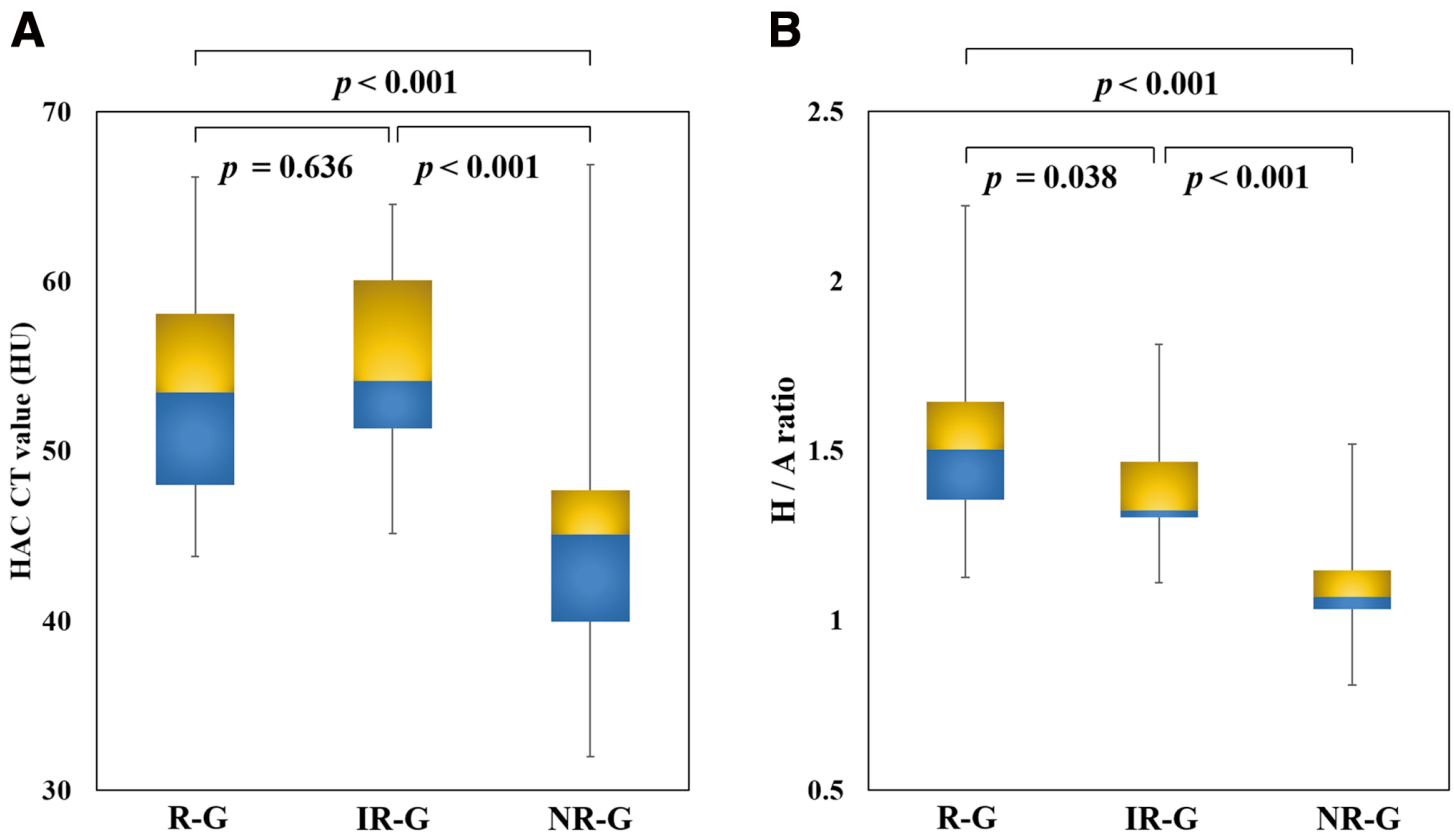

Figure 3. (A) Comparison of the CT attenuation values of the HAC sign. A box plot showing the comparison of CT attenuation values of the HAC sign among the R-G, IR-G, and NR-G. The CT attenuation values of the HAC sign in the R-G and IR-G were significantly higher than that in the NR-G (R-G: 53.7 HU; IR-G: $55.5 \mathrm{HU}$; NR-G: $45.4 \mathrm{HU}$; 1-way analysis of variance with Tukey's honestly significant difference test) (B) Comparison of the $H / A$ ratios. A box plot showing the comparison of $H / A$ ratios among the R-G, IR-G, and NR-G. The H/A ratios in the R-G and IR-G were significantly higher than that in the NR-G (R-G: 1.54; IR-G: 1.38; NR-G: 1.11; 1-way analysis of variance with Tukey's honestly significant difference test). A, computed tomography attenuation value of the aortic lumen; $\mathrm{CT}$, computed tomography; $\mathrm{H}$, computed tomography attenuation value of the high-attenuating crescent sign; HAC, high-attenuating crescent; HU, Hounsfield unit; IR-G, impending rupture group; NR-G, non-rupture group; R-G, rupture group.

ity of the HAC sign as a rupture/impending rupture indicator was $76.4 \%(52 / 68)$, whereas it was $94.1 \%(16 / 17)$ as an impending rupture indicator.

We excluded 16 patients with rupture/impending rupture without a HAC sign (15/1) to evaluate the HAC sign. Consequently, the study enrolled 76 patients (rupture, $\mathrm{n}=36$; impending rupture, $\mathrm{n}=16$; and non-rupture, $\mathrm{n}=24$ ) with AA with a HAC sign. The clinical characteristics of the participants are summarized in Table 1 . The mean age of the patients was 77.0 \pm 9.9 years (range, 52-98 years). Fifty-three patients were males and 23 females. Twentyeight $(36.8 \%)$ and $48(63.2 \%)$ AAs were thoracic and abdominal, respectively. Sixty-one (80.3\%) and $15(19.7 \%)$ AAs were of the fusiform and saccular types, respectively. The mean aneurysmal diameter of the maximum minor axis was $62.0 \pm 14.8 \mathrm{~mm}$ (range, $36.0-89.9 \mathrm{~mm}$ ).

\section{Comparison of the $\mathrm{H}$ and $\mathrm{H} / \mathrm{A}$ Ratio}

Table 2 shows the comparison of patient characteristics among the 3 groups. Table 3 shows the H, A, and the H/A ratio for the R-G, IR-G, and NR-G (Figure 2). The $\mathrm{H}$ and the H/A ratio were significantly higher in the $R-G$ and IR-G than those in the NR-G $(\mathrm{P}<0.001$, Figure 3A; $\mathrm{P}<0.001$, Figure 3B, respectively). Furthermore, although there was no significant difference between the R-G and IR-G regarding the $H(P=0.636$, Figure $3 \mathbf{A})$, the $H / A$ ratio was significantly higher in the $R-G$ than in the IR-G $(P=0.038$, Figure 3B). The distribution of the HAC CT value, and that of the H/A ratio, is presented in Supplementary Figure.

\section{Cut-Off Values for the $\mathrm{H}$ and the H/A Ratio}

Area under the curve (AUC) analysis was performed to compare the $\mathrm{H}$ and the $\mathrm{H} / \mathrm{A}$ ratio between the IR-G and NR-G. According to the ROC analysis, the optimal cut-off value for the $\mathrm{H}$ was $50.3 \mathrm{HU}$, with an AUC of 0.875 (sensitivity, $87.5 \%$; specificity, $87.5 \%$ ) and that for the H/A ratio was 1.3, with an AUC of 0.909 (sensitivity, 91.7\%; specificity, $87.5 \%$ ) (Figure 4). Thus, $\mathrm{H}>50.3 \mathrm{HU}$ or a H/A ratio $>1.3$ denoted a higher possibility of impending rupture than of non-rupture.

\section{Discussion}

In our study, the sensitivities of the HAC sign as a rupture or impending rupture indicator were high and consistent with those reported in a previous study (77\%). ${ }^{1}$ Additionally, our study demonstrated that in AAs with a HAC sign, the $\mathrm{H}$ and the H/A ratio were significantly higher in the R-G and IR-G than in the NR-G. These results indicated that the HAC sign in cases of rupture or impending rupture of an AA may involve relatively acute hemorrhage in the mural thrombus or aneurysmal wall compared with those of non-rupture of the AA. Conversely, the HAC sign in cases of non-rupture of an AA might demonstrate chronic hemorrhage. In addition, another potential reason of the HAC sign in non-rupture cases is the heterogeneity 
A

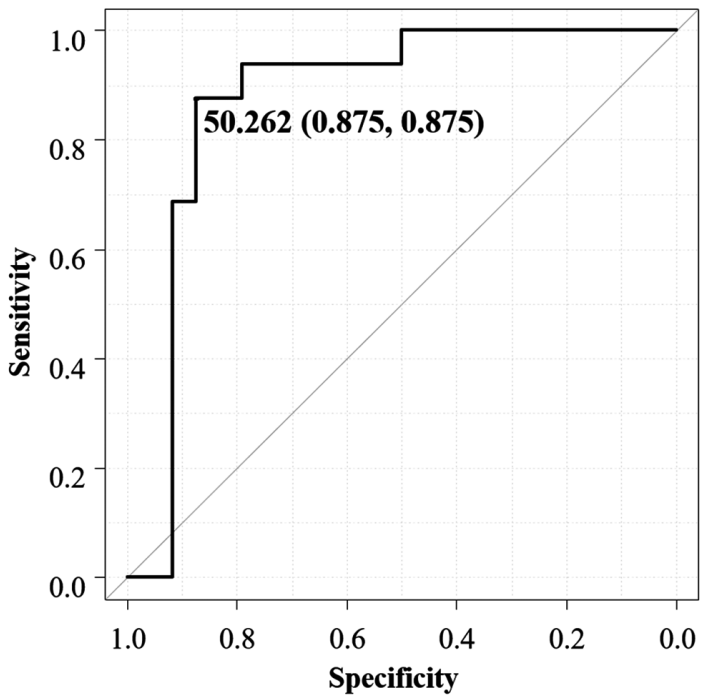

B

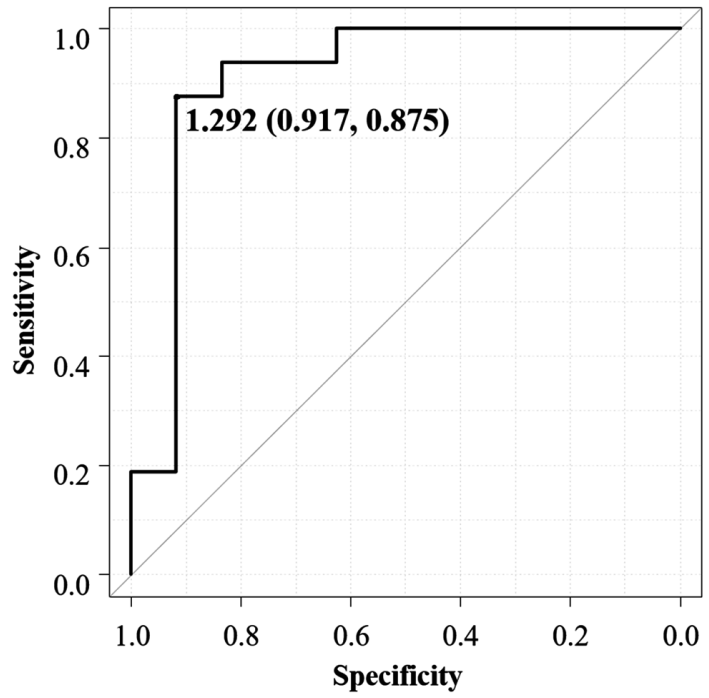

Figure 4. Receiver operator characteristic analysis in the impending rupture group and non-rupture group. (A) Computed tomography attenuation value of the high-attenuating crescent sign. (B) Computed tomography attenuation value of the high-attenuating crescent sign/computed tomography attenuation value of the aortic lumen ratio.

of the attenuation of the mural thrombus. The relatively high density area compared to the decreased density area of the mural thrombus may cause misunderstanding concerning the HAC sign.

The A in the R-G was significantly lower than those in the IR-G and NR-G, probably because the A depends on the hemoglobin levels. Collins et al found a significant correlation between the hemoglobin levels and the derived aortic attenuation value. ${ }^{20}$ In our study, the hemoglobin levels were significantly lower in the R-G than in the IR-G and NR-G. Interestingly, although there was no significant difference in the $\mathrm{H}(\mathrm{P}=0.636)$ between the $\mathrm{R}-\mathrm{G}$ and $\mathrm{IR}-\mathrm{G}$, the $H / A$ ratio was significantly higher in the $R-G$ than in the NR-G $(P=0.038)$, probably because the $A$ in the $R-G$ was significantly lower than those in the IR-G and NR-G.

Our results showed that the $\mathrm{H}$ and $\mathrm{H} / \mathrm{A}$ ratio, with cutoff values of $50.3 \mathrm{HU}$ and 1.3 , respectively, may be sensitive predictive factors in patients with impending rupture of AA. In the ROC analysis, our findings indicated that the $\mathrm{H}$ and H/A ratio could be useful predictive markers for distinguishing impending rupture from non-rupture. When the $\mathrm{H}$ is $>50.3 \mathrm{HU}$ or the $\mathrm{H} / \mathrm{A}$ ratio is $>1.3$, there is a higher possibility that the AA could belong to the IR-G than to the NR-G. Our results showed that the accuracy of the cut-off value of the H/A ratio was higher than that of the $H$.

Our study had several limitations. First, we used several types of multidetector CT scanners, from a 16-row multidetector to a 320-row multidetector, which might have caused selection bias. Second, the HAC sign was defined by the attenuation being higher area in the aortic wall or the mural thromubus than that of the aortic lumen on unenhanced CT and determined by visual inspection.

In conclusion, the $\mathrm{H}$ and the $\mathrm{H} / \mathrm{A}$ ratio were significantly higher in cases with rupture or impending rupture of AA than in those with non-rupture of AA. Hence, it may be useful to measure the $\mathrm{H}$ and the $\mathrm{H} / \mathrm{A}$ ratio to distinguish impending rupture from non-rupture cases.

\section{Acknowledgments}

None.

\section{Sources of Funding}

This research did not receive any specific grant from funding agencies in the public, commercial, or not-for-profit sectors.

\section{Disclosures}

The authors declare that there are no conflicts of interest.

\section{Ethical Statement}

All procedures performed in studies involving human participants were in accordance with the ethical standards of the institutional and/or national research committee and with the 1964 Helsinki Declaration and its later amendments or comparable ethical standards. The study protocols of this retrospective analysis were approved by the Nippon Medical School Hospital Institutional Review Board (Protocol Identification Number: B-2020-149). The requirement for informed consent was waived because of the retrospective nature of the study.

\section{Data Availability}

The deidentified participant data will not be shared.

\section{References}

1. Mehard WB, Heiken JP, Sicard GA. High-attenuating crescent in abdominal aortic aneurysm wall at CT: A sign of acute or impending rupture. Radiology 1994; 192: 359-362.

2. Siegel CL, Cohan RH, Korobkin M, Alpern MB, Courneya DL, Leder RA. Abdominal aortic aneurysm morphology: CT features in patients with ruptured and nonruptured aneurysms. $A J R \mathrm{Am}$ J Roentgenol 1994; 163: 1123-1129.

3. Schwartz SA, Taljanovic MS, Smyth S, O'Brien MJ, Rogers LF. CT findings of rupture, impending rupture, and contained rupture of abdominal aortic aneurysms. AJR Am J Roentgenol 2007; 188: W57-W62.

4. Pillari G, Chang JB, Zito J, Cohen JR, Gersten K, Rizzo A, et 
al. Computed tomography of abdominal aortic aneurysm: An in vivo pathological report with a note on dynamic predictors. Arch Surg 1988; 123: 727-732.

5. Gonsalves CF. The hyperattenuating crescent sign. Radiology 1999; 211: 37-38.

6. Rakita D, Newatia A, Hines JJ, Siegel DN, Friedman B. Spectrum of CT findings in rupture and impending rupture of abdominal aortic aneurysms. Radiographics 2007; 27: 497-507.

7. Taheri MS, Haghighatkhah H, Pourghorban R, Hosseini A. Multidetector computed tomography findings of abdominal aortic aneurysm and its complications: A pictorial review. Emerg Radiol 2013; 20: 443-451

8. Cerna M, Kocher M, Thomas RP. Acute aorta, overview of acute CT findings and endovascular treatment options. Biomed Pap Med Fac Univ Palacky Olomouc Czech Repub 2017; 161: 14-23.

9. Wadgaonkar AD, Black JH 3rd, Weihe EK, Zimmerman SL, Fishman EK, Johnson PT. Abdominal aortic aneurysms revisited: MDCT with multiplanar reconstructions for identifying indicators of instability in the pre- and postoperative patient. Radiographics 2015; 35: 254-268.

10. Gish DS, Baer JA, Crabtree GS, Shaikh B, Fareedy SB. Impending aortic aneurysm rupture: A case report and review of the warning signs. J Community Hosp Intern Med Perspect 2016; 6: 32217.

11. Pillari GP. Crescent sign origin and the thrombus-to-lumen ratio in abdominal aortic aneurysm. Radiology 2000; 214: 604

12. Litmanovich D, Bankier AA, Cantin L, Raptopoulos V, Boiselle PM. CT and MRI in diseases of the aorta. AJR Am J Roentgenol 2009; 193: $928-940$.

13. Khan S, Verma V, Verma S, Polzer S, Jha S. Assessing the potential risk of rupture of abdominal aortic aneurysms. Clin Radiol
2015; 70: 11-20.

14. Agarwal PP, Chughtai A, Matzinger FR, Kazerooni EA. Multidetector CT of thoracic aortic aneurysms. Radiographics 2009; 29: $537-552$.

15. Vu KN, Kaitoukov Y, Morin-Roy F, Kauffmann C, Giroux MF, Thérasse E, et al. Rupture signs on computed tomography, treatment, and outcome of abdominal aortic aneurysms. Insights Imaging 2014; 5: 281-293.

16. Ahmed MZ, Ling L, Ettles DF. Common and uncommon CT findings in rupture and impending rupture of abdominal aortic aneurysms. Clin Radiol 2013; 68: 962-971.

17. Arita T, Matsunaga N, Takano K, Nagaoka S, Nakamura H, Katayama S, et al. Abdominal aortic aneurysm: Rupture associated with the high-attenuating crescent sign. Radiology 1997; 204: $765-768$.

18. Roy J, Labruto F, Beckman MO, Danielson J, Johansson G, Swedenborg J. Bleeding into the intraluminal thrombus in abdominal aortic aneurysms is associated with rupture. $J$ Vasc Surg 2008; 48: $1108-1113$.

19. Boules TN, Compton CN, Stanziale SF, Sheehan MK, Dillavou ED, Gupta N, et al. Can computed tomography scan findings predict "impending" aneurysm rupture? Vasc Endovascular Surg 2006; 40: $41-47$.

20. Collins AJ, Gillespie S, Kelly BE. Can computed tomography identify patients with anaemia? Ulster Med $J$ 2001; 70: 116-118.

\section{Supplementary Files}

Please find supplementary file(s);

http://dx.doi.org/10.1253/circj.CJ-21-0541 